\title{
Thermoelastic effect in liquid mercury at high hydrostatic pressures
}

ERNESTO L. RODRIGUEZ

Owens-Corning Fiberglas Corporation, Technical Center, Granville, Ohio 43023, USA

FRANK E. FILISKO

Department of Materials and Metallurgical Engineering, The University of Michigan, Ann Arbor, Michigan 48109, USA

The rapid deformation of liquid and solid materials usually results in temperature changes. This rapid deformation is considered to be a nearly adiabatic process where the heat generated during the deformation changes the temperature of the material. Early experiments showed that metals cooled down on sudden stretching and warmed up upon compression [1]. In 1805, Gough [2] observed that the temperature of a rubber band increased when it was suddenly stretched and decreased when the force applied was released. These reversible thermomechanical effects, also defined as the thermoelastic effect, were later widely studied by Thomson (Lord Kelvin) [3]. In 1853, Thomson [4] proposed a general thermodynamic formula that described the change in temperature as the result of the rapid application of pressure on fluids. This Thomson equation can be written as

$$
\frac{\partial T}{\partial P}=\frac{T_{0} \alpha_{\mathrm{v}}}{\varrho C_{\mathrm{p}}}
$$

where $(\partial T / \partial P)$ is the thermoelastic coefficient which describes the differential in temperature or temperature change as the result of an external differential in pressure or a pressure change, $T_{0}$ is the reference temperature, $\alpha_{\mathrm{v}}$ is the volume thermal expansion coefficient, $\varrho$ is the density, and $C_{\mathrm{p}}$ is the heat capacity.

Joule $[5,6]$ verified the Thomson equation by measuring the temperature changes produced by suddenly stretching or compressing uniaxially at low stresses a variety of materials, including mercury. Since then, no other studies have been reported on the thermoelastic effect in liquid mercury. The present work reports thermal effects in liquid mercury using hydrostatic pressures from 13 to $200 \mathrm{MN} \mathrm{m}^{2}$ and over the temperature range 298 to $370 \mathrm{~K}$.

Fig. 1 illustrates schematically the experimental set-up including the high-pressure pump and equipment. A $1 \mathrm{hp}$ standard air compressor with a regulated output variable to $100 \mathrm{psi}\left(0.69 \mathrm{MN} \mathrm{m}^{-2}\right)$ was used to drive the air-driven high-pressure reciprocating pump (Teledyne Sprague). This pump also attenuated the pressure by a factor of 300 to a maximum of 30000 psi $\left(200 \mathrm{MN} \mathrm{m}^{-2}\right)$. The desired pressure was selected by a regulator located between the compressor and the high-pressure pump. The pressure was measured by two Bourdon gauges, each with a maximum of $50000 \mathrm{psi}$ and sensitivity of $500 \mathrm{psi}$ per division. One junction of an iron-constantan thermo-

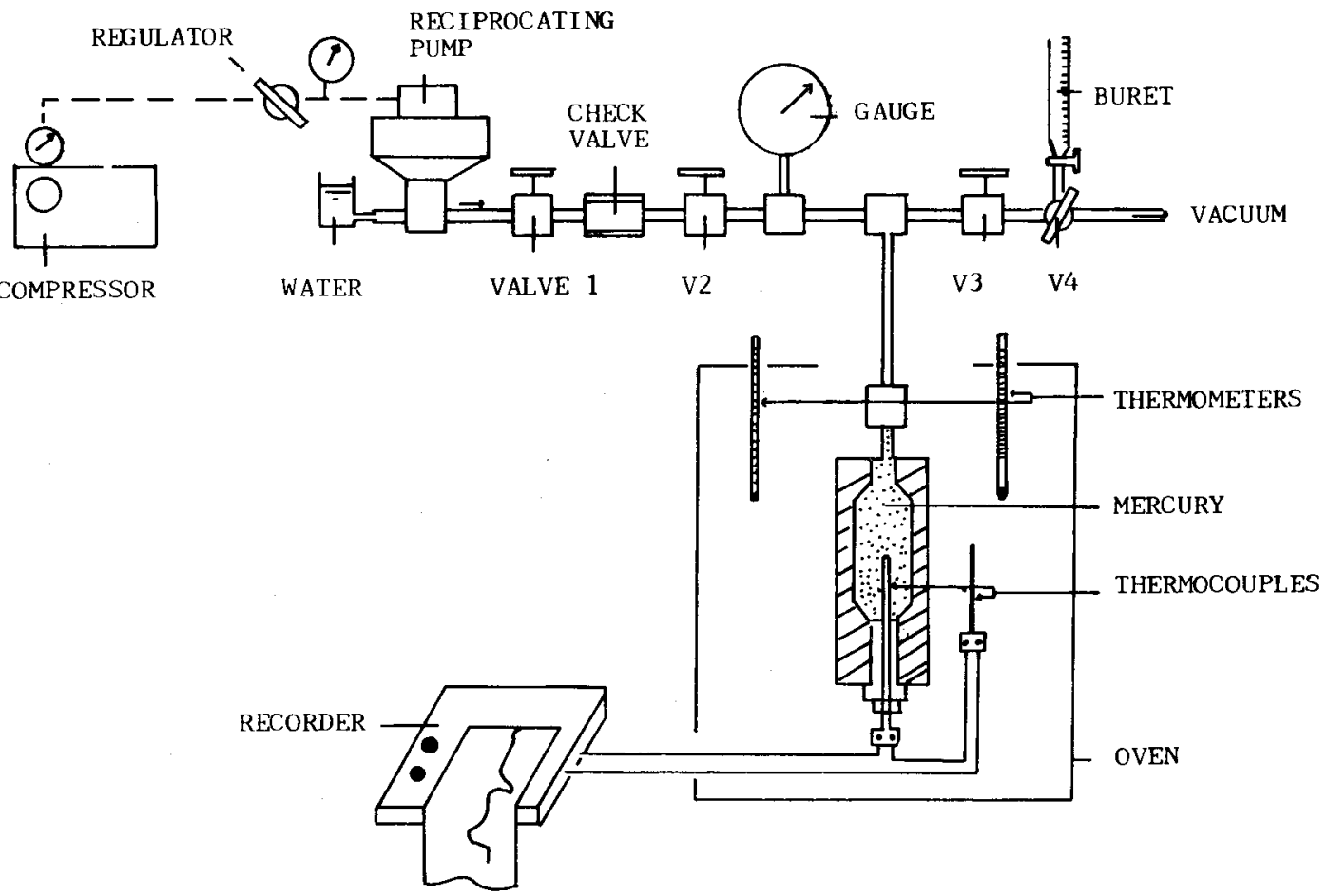

Figure 1 The high-pressure system. 


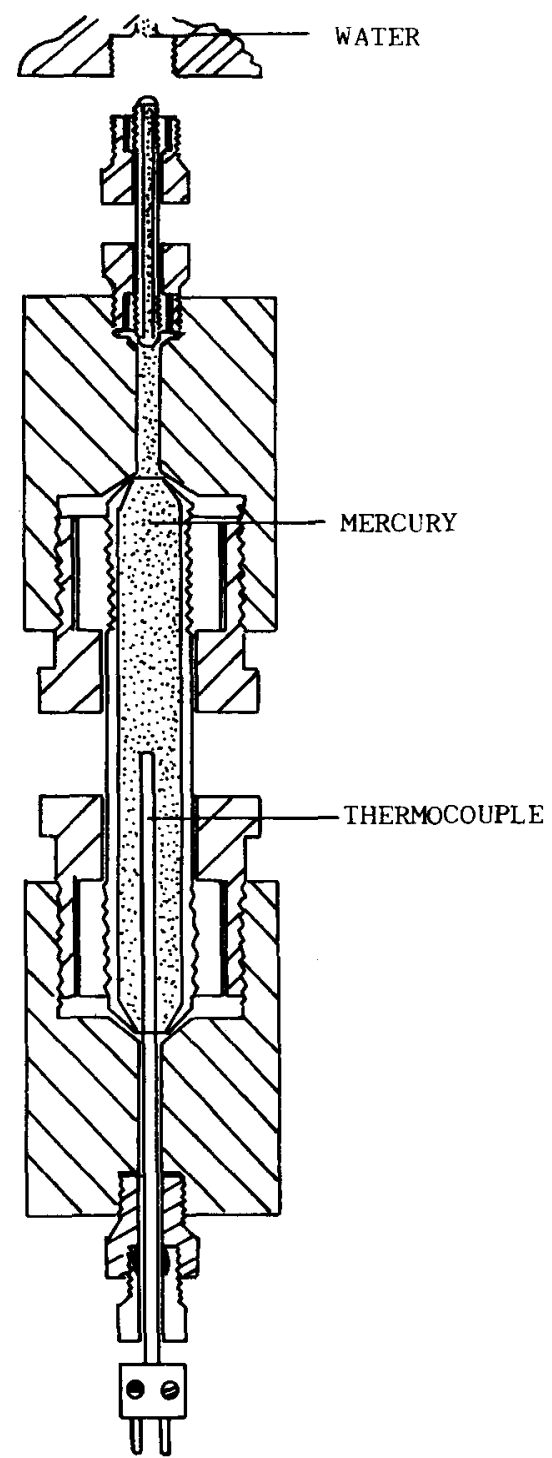

Figure $2 \mathrm{~A}$ cross-section of the high-pressure unit.

couple inside a $0.062 \mathrm{in} .(1.57 \mathrm{~mm})$ diameter stainless steel sheath and mercury were located inside the highpressure cell, as illustrated in Fig. 2. With this arrangement, the high-pressure unit can easily be removed and reconnected to the high-pressure line. Water was used as the transmitting fluid throughout the system with the exception of the high-pressure cell. The oven temperature was controlled to better than $\pm 0.5^{\circ} \mathrm{C}$.

The second junction of the $\Delta T$ measuring thermocouple was located outside the high-pressure unit but was located in direct thermal contact with it. This arrangement allowed us to measure directly only the temperature difference generated by the pressure variations, and the maximum sensitivity, since both junctions were at atmospheric pressure, and the output of the thermocouple was virtually zero. The sensitivity of the $\Delta T$ measuring system with the recorder of the $1 \mathrm{mV}$ scale was about $\pm 0.1^{\circ} \mathrm{C}$. Mercury was obtained from a commercial source and used asreceived.

A specific pressure was built up behind valve 2 (see Fig. 1). The rapid opening of valve 2 caused pressure to be transmitted almost instantaneously to the mercury (a "pressure jet"). The pressure was released

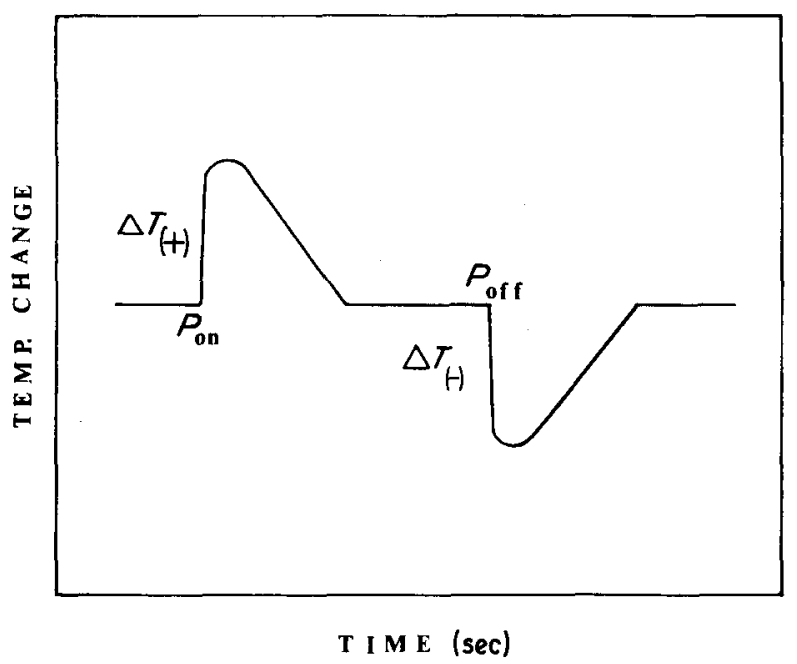

Figure 3 Representation of the thermoelastic effect corresponding to a delayed release of pressure.

by the rapid opening of valve 3 to atmospheric pressure. Fig. 3 shows a typical recording of the thermoelastic effect. In the figure, we observe the following steps.

1. From A to B represents the initial state where $P_{0}$ is the atmospheric pressure, and $T_{0}$ is the reference temperature or temperature of the oven.

2. At B a pressure is applied, $P_{\mathrm{on}}$.

3. From $B$ to $C$ the mercury warms up. The increase in temperature $\Delta t_{(+)}$is recorded.

4. From $C$ to $D$ the mercury relaxes until it reaches approximately the initial temperature $T_{0}$.

5. At $E$ the pressure is rapidly released.

6. From $\mathrm{E}$ to $\mathrm{F}$ the sample cools down by a $\Delta T_{(-)}$ amount. From $F$ to $G$ the mercury returns to the original conditions $P_{0}$ and $T_{0}$.

7. A complete reversibility $\Delta t_{(+)}=\Delta t_{(-)}$was observed throughout the experiment.

Fig. 4 shows the average temperature changes as a function of the applied pressure for three temperatures. It was found that increasing the pressure and the reference temperature resulted in an increase in the temperature change. For example, for a rapid hydrostatic pressure of $110 \mathrm{MN} \mathrm{m}^{-2}$, the temperature changes of $\Delta T=2.52^{\circ} \mathrm{C}, \Delta T=2.95^{\circ} \mathrm{C}, \quad$ and $\Delta T=3.21^{\circ} \mathrm{C}$ were measured for the reference temperatures 297,336 , and $368 \mathrm{~K}$, respectively.

A curve analysis showed a linear dependence of the temperature change with pressure $\Delta P$. The results of the analysis are shown in Table I. If $\Delta T=T-T_{0}$, and differentiating on both sides, we obtain $\partial \Delta T \cong$ $\mathrm{d} T$. Also, if $\Delta P=P-P_{0}$, where $P_{0}=1 \mathrm{~atm}$, we obtain $\mathrm{d} \Delta P \cong \mathrm{d} P$. Thus, the slope of the linear plot of $\Delta T$ against $\Delta P$ is approximately $(\partial T / \partial P)$, the experimental thermoelastic coefficient.

TABLE I Curve-fitting analysis results for mercury

\begin{tabular}{lll}
\hline $\begin{array}{l}\text { Temperature } \\
\text { (K) }\end{array}$ & $y=a+b x$ & $\begin{array}{l}\text { Coefficient of } \\
\text { determination, } \\
R^{2}\end{array}$ \\
\hline 297 & $y=0.0981+0.0218 x$ & 0.999 \\
336 & $y=0.1385+0.0255 x$ & 1.000 \\
368 & $y=0.1450+0.0280 x$ & 0.999 \\
\hline
\end{tabular}




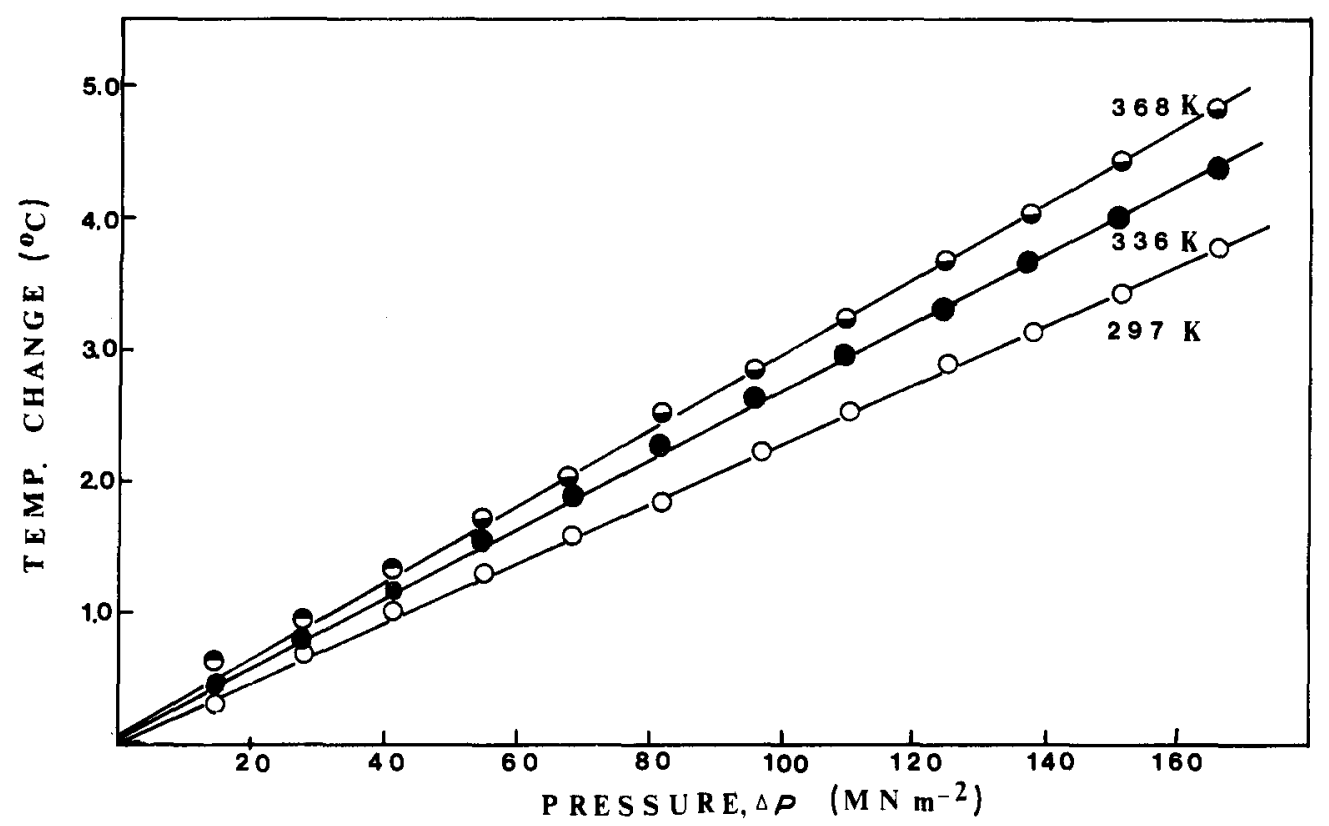

Figure 4 Temperature changes as a function of applied pressure for mercury at different temperatures.

Vokalovich and Fokin [7] reported the thermal expansion coefficient, the density, and the heat capacity of mercury. For our study, the physical values selected from the Vokalovich and Fokin data are given in Table II. From Equation 1, the predicted thermoelastic coefficients $(\partial T / \partial P)$ could be estimated and compared with the coefficients obtained from the curve-fitting analysis. These results are shown in Table III. A $20 \%$ difference between the predicted and the experimental thermoelastic coefficients was calculated. Fig. 5 shows the predicted and experimental thermoelastic coefficient values as a function of the reference temperature, where a linear dependence was also observed.

In spite of the differences found between the experimental and predicted thermoelastic coefficient, the Thomson equation is still valid and useful for estimating the thermal effect in mercury upon rapid deformation. From thermodynamics variables, it is frequently assumed that the coefficient $\alpha_{v} \varrho / C_{\mathrm{p}}$ in Equation 1 is not a function of pressure and temperature, so that Equation 1 can be integrated within reasonable limits. This assumption is valid and holds very well at very low stresses or pressures. However, when relatively large stresses or pressures are applied, considerable deviations may occur between the predicted and experimental data.

The thermal effect in mercury occurred very rapidly. The mercury warmed up instantaneously upon the rapid application of pressure. After reaching the maximum temperature, it gave out heat to the surroundings, as is illustrated in Fig. 3 from the path

TABLE II Physical data for mercury (from [7])

\begin{tabular}{llll}
\hline $\begin{array}{l}\text { Temperature } \\
(\mathrm{K})\end{array}$ & $\begin{array}{l}\varrho \\
\left(\mathrm{kg} \mathrm{m}^{-3}\right)\end{array}$ & $\begin{array}{l}\alpha_{\mathrm{v}} \\
\left(\mathrm{K}^{-1}\right)\end{array}$ & $\begin{array}{l}C_{\mathrm{p}} \\
\left(\mathrm{J} \mathrm{kg}^{-10} \mathrm{C}^{-1}\right)\end{array}$ \\
\hline 297. & $13.53640 \times 10^{3}$ & $1.81103 \times 10^{-4}$ & $0.1396 \times 10^{3}$ \\
336 & $13.43606 \times 10^{3}$ & $1.80615 \times 10^{-4}$ & $0.1381 \times 10^{3}$ \\
368 & $13.36348 \times 10^{3}$ & $1.80518 \times 10^{-4}$ & $0.1373 \times 10^{3}$ \\
\hline
\end{tabular}

$\mathrm{C}$ to E. Mercury showed that cooling during the rapid release of pressure reached the minimum temperature and then absorbed heat from the surroundings to return to the original condition of $T_{0}$ and $1 \mathrm{~atm}$. In Fig. 3, the trajectory from applying the pressure (point B) to reaching the initial temperature (point D) took approximately $6 \mathrm{sec}$. This rapid absorption and dissipation of heat is related to the high thermal conductivity of mercury.

Müller [8] reviewed the thermodynamics of deformation including the reversible thermal effects that take place in any material upon rapid deformation. Müller stated that the elastic restoring force of a material is usually considered to be the result of attractive forces between atoms and molecules. After deformation these forces tend to bring the molecular structure back to its original equilibrium position. These molecular forces may be of entropic or enthalpic nature. For example, purely entropic elastic behaviour occurs in ideal gases and also in ideal rubbers. For these, the free energy, $F=U-T S$, is determined by $S$, the entropy. By definition, $(\partial U / \partial V)_{T}=0$ for an ideal gas and $(\partial U / \partial l)_{T}=0$ for an ideal rubber. For these cases, the thermal effects resulting from rapid deformation are associated with the entropic changes in the materials, the internal energy $(U)$ being virtually zero.

In crystalline and metallic solids, it is customary to define these materials as enthalpically elastic. The term $(\partial U / \partial l)_{T}$ is assumed to describe the restoring

TABLE III Thermoelastic coefficient ( $\partial T / \partial P)$ values for mercury

\begin{tabular}{lll}
\hline $\begin{array}{l}\text { Temperature } \\
(\mathrm{K})\end{array}$ & $\begin{array}{l}\text { Experimental values* } \\
\left({ }^{\circ} \mathrm{C} \mathrm{m}^{2} \mathrm{MN}^{-1}\right)\end{array}$ & $\begin{array}{l}\text { Predicted values } \\
\left({ }^{\circ} \mathrm{C} \mathrm{m}^{2} \mathrm{MN}^{-1}\right)\end{array}$ \\
\hline 297 & 0.0218 & 0.0285 \\
336 & 0.0255 & 0.0327 \\
368 & 0.0280 & 0.0362 \\
\hline
\end{tabular}

${ }^{*}$ From slope of $\Delta T$ against $\Delta P$.

$\dagger$ From the equation $(\partial T / \partial P)=\alpha_{\mathrm{v}} T_{0} / \varrho C_{\mathrm{p}}$. 


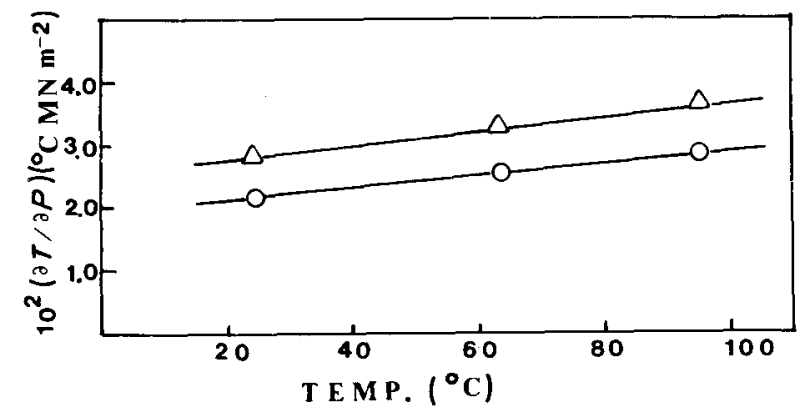

Figure 5 Thermoelastic coefficients $(\partial T / \partial P)$ as a function of temperature. Experimental values $(O)$ determined from the slope of $\Delta T$ against $\Delta P$ and the predicted values $(\Delta)$ determined from Equation 1.

forces of the materials which are closely associated to the changes in the molecular distances between atoms and molecules.

These two extremes are obviously not exact and are in need of experimental tests. It is most likely that both the entropy and the internal energy contribute to the elastic restoring forces. In our specific case, the thermoelastic effect observed during rapid compression and decompression in mercury may be associated with the changes in the molecular organization (entropic) as well as the changes in the molecular distance between atoms (enthalpic).

\section{References}

1. W. WEBER, Prog. Ann. Phys. 20 (1830) 177.

2. J. GOUCH, Proc. Lit. Phil. Soc. Manchester (2nd ser.), I (1805) 288

3. W. THOMSON (Lord Kelvin), "Mathematical and Physical Papers", Vol. 1 (Cambridge, London, 1882).

4. Idem, "Mathematical and Physical Papers", Vol. 3 (Cambridge, London 1890) pp. 236-239.

5. J. P. JOULE, Proc. R. Soc. 8 (1857) 355.

6. Idem, Phil. Trans. 149 (1859) 91.

7. M. P. VOKALOVICH and R. V. FOKIN, "Thermophysical Properties of Mercury" (Standard Press, 1972).

8. F. H. MULLER, "Rheology: Theory and Applications", edited by F. R. Eirich, Vol. 5 (Academic Press, New York and London, 1969) pp. 417-489.

Received 1 July

and accepted 15 July 1985 